\title{
La institucionalización del campo académico de la comunicación en México y en Brasil. Un primer acercamiento comparativo
}

Raúl Fuentes Navarro Instituto Tecnológico y de Estudios Superiores de Occidente (ITESO)

A

FINES de 1988 se puso en marcha un proyecto de investigación sin precedentes en el campo académico de la comunicación en América Latina: el Estudio Comparativo de los Sistemas de Comunicación Social en Brasil y en México, impulsado por José Marques de Melo y promovido por la Sociedade Brasileira de Estudios Interdisciplinarios da Comunicaçao (INTERCOM) y el Consejo Nacional para la Enseñanza y la Investigación de las Ciencias de la Comunicación (CONEICC). Además de fomentar el acercamiento e intercambio académico personal e institucional de diez investigadores de cada país.

La finalidad principal de este estudio es detectar las semejanzas y contrastes entre los sistemas de comunicación social operantes en Brasil y México. Se pretende construir perfiles comparativos de algunos de los elementos que constituyen cada sistema, para constatar evidencias sobre su desarrollo histórico y su configuración actual. La primera intención de ese diagnóstico es conocer las respectivas particularidades tecnologicas, económicas, jurídicas, políticas y socioculturales, como punto de partida para análisis más profundos sobre las implicaciones coyunturales y estructurales de las identidades $y$ las diferencias descritas (INTERCOM, 1988).

Entre los diez subsistemas propuestos para el análisis, el proyecto incluyó dos específicamente académicos: el de la Enseñanza de la Comunicación (encargado al brasileño Antonio Carlos de Jesús y al mexicano Carlos E. Luna Cortés) y el de la Investigación de la Comunicación (con María Immacolata Vasallo de Lopes y Raúl Fuentes Navarro como responsables). El proyecto general, metodológicamente planteado como una "investigación descriptiva, basada en información secundaria", previó tres etapas: una revisión sistemática de la bibliografía existente 
sobre cada subsistema en cada país, la elaboración de un "perfil descriptivo del trayecto histórico y el estado actual" de cada subsistema nacional y, finalmente, un análisis comparativo. Dadas las diferencias de enfoque y los diversos ritmos con los que se trabajó, cuatro años y medio después del inicio en ninguno de los diez subsistemas se ha llegado a la tercera etapa, aunque hay un buen número de productos parciales terminados, muchos de ellos artículos y libros ya publicados, y varios procesos de intercambio satisfactoriamente establecidos.

En rigor, este artículo no pretende la comparación prevista en el proyecto binacional entre la investigación de la comunicación en Brasil y en México, por las disparidades en grado de avance y del método utilizado, y porque es realizado desde el punto de vista de uno solo de los responsables, mucho más en función de su investigación actual (Determinaciones Socioculturales del Campo Académico de la Comunicación en México) que del proyecto referido. No obstante, busca desde otro marco aprovechar los aportes de los colegas comprometidos en el análisis comparativo de la enseñanza y la investigación en ambos paíscs y eventualmente animar la continuación actualizada de la búsqueda compartida.

\section{Dos países grandes con problemas enormes: un primer contexto}

Por su tamaño y nivel de desarrollo, Brasil y México son indudablemente las naciones latinoamericanas que con mayor claridad manifiestan las condiciones que el orden económico y político mundial vigente ha impuesto a los países periféricos. Ubicados geográficamente y caracterizados en muchos aspectos como dos de los "extremos" de América Latina, en ambos países (alguna vez imperios frustrados) se hacen evidentes las contradicciones entre la miseria y la riqueza extremas, entre las tradiciones culturales más profundas y el afán de insertarse en una modernidad de "primer mundo". Sin entrar a esta escala en la descripción sistemática y menos en el análisis estructural, datos de la Encyclopaedia Britannica (1990) nos permiten decir que los dos países suman más de la mitad de la extensión territorial y de la población de América Latina; que sus Productos Nacionales Brutos sumados representan dos tercios del correspondiente a los veintiún países del área y sus deudas externas más de la mitad. Sin embargo, hay que distinguir: el territorio de Brasil (quinto en extensión el mundo) es cuatro y media veces mayor que el de México (decimocuarto); su población (sexta mayor en el mundo) es casi el doble 
que la mexicana (undécima); su Producto Nacional Bruto es más de dos veces mayor que el nuestro, aunque las deudas externas son prácticamente iguales. El PNB per cápita es de 2,550 dólares al año para Brasil y 1,990 para México. Pero ninguno de los dos países puede estar conforme con la distribución interna de su riqueza: aunque los datos corresponden a 1988 para Brasil y a 1983 para México, el $20 \%$ más rico de la población acumula el $66.4 \%$ del ingreso en Brasil y el $50.6 \%$ en México, mientras que el $20 \%$ más pobre de la población recibe apenas el $2.4 \%$ del ingreso en el primer país y el $4 \%$ en el segundo.

Para nuestros propósitos, las cifras sobre los respectivos sistemas educativos son quizá las más elocuentes. Aunque en ambos casos se trata de índices propios de países francamente subdesarrollados, el porcentaje de la población mexicana mayor de 25 años con educación postsecundaria es un poco mayor que el brasileño: $5.3 \%$ y $5.0 \%$ respectivamente. De igual manera, el porcentaje de alfabetizados entre los mayores de 15 años es mayor en México que en Brasil: 87.3\% contra 81.1\%. Mientras que Brasil tiene 1,041 estudiantes de educación superior por cada 100,000 habitantes, México tiene 1,543. Finalmente, Brasil dedica el 4.5\% del PNB como gasto público en educación y México el 5.3\% Los datos más elementales de los sistemas educativos nacionales son los siguientes:

Tabla 1. Sistemas educativos en Brasil y México

\begin{tabular}{|c|c|c|c|c|c|c|}
\hline \multirow{2}{*}{$\begin{array}{l}\text { NIVEL } \\
\text { Primaria }\end{array}$} & \multicolumn{2}{|c|}{ ESCUELAS } & \multicolumn{2}{|c|}{ Profesores } & \multicolumn{2}{|c|}{ EsTUDIANTES } \\
\hline & $\begin{array}{l}\text { B: } \\
\text { M: }\end{array}$ & $\begin{array}{r}201,541 \\
83,924\end{array}$ & & $\begin{array}{r}1 ' 119,907 \\
476,718\end{array}$ & $\begin{array}{l}\text { B: } \\
\text { M: }\end{array}$ & $\begin{array}{l}26^{\prime} 821,134 \\
14^{\prime} 585,300\end{array}$ \\
\hline Secundaria & $\begin{array}{l}\text { B: } \\
\text { M: }\end{array}$ & $\begin{array}{l}10,244 \\
30,173\end{array}$ & $\begin{array}{l}\text { B: } \\
\text { M: }\end{array}$ & $\begin{array}{l}229,183 \\
416,178\end{array}$ & $\begin{array}{l}\text { B: } \\
\text { M: }\end{array}$ & $\begin{array}{l}3^{\prime} 339,090 \\
7^{\prime} 109,700\end{array}$ \\
\hline Superior & $\begin{array}{l}\text { B: } \\
\text { M: }\end{array}$ & $\begin{array}{r}871 \\
1,273\end{array}$ & $\begin{array}{l}\text { B: } \\
\text { M: }\end{array}$ & $\begin{array}{l}125,412 \\
112,763\end{array}$ & $\begin{array}{l}\text { B: } \\
\text { M: }\end{array}$ & $\begin{array}{l}1^{\prime} 503,560 \\
1^{\prime} 124,100\end{array}$ \\
\hline
\end{tabular}

Por lo que toca a los sistemas de comunicación, sin duda el brasileño y el mexicano son los más desarrollados de América Latina y sus respectivas empresas "líderes" (O Globo y Televisa) además de tener historias y estructuras semejantes, se han expandido internacionalmente de manera notable. Datos de 1988, consignados por 
Tabla 2. Sistemas de comunicación en Brasil y México

\begin{tabular}{|c|c|c|c|c|c|c|}
\hline \multirow{2}{*}{$\begin{array}{l}\text { DIARIOS } \\
\text { B: } 279\end{array}$} & \multicolumn{2}{|c|}{ EMISORAS DE RADIO } & \multicolumn{2}{|c|}{ EMISORAS DE TV } & \multicolumn{2}{|c|}{ SALAS DE CINE } \\
\hline & B: & 1,729 & B: & 137 & B: & 1,403 \\
\hline M: 392 & M: & 887 & $\mathbf{M}:$ & 430 & M: & 1,775 \\
\hline
\end{tabular}

la Encyclopaedia Britannica (1990), dan una idea elemental, suficiente para los propósitos presentes, de la magnitud de los sistemas.

Excepto en radio y en recepción de televisión, el sistema mexicano es mayor que el brasileño. Algunos datos complementarios permiten matizar esta impresión: en cuanto a los diarios, el índice de circulación por mil habitantes pone a México, con 142 ejemplares, en tercer lugar de América Latina detrás de Puerto Rico (183) y Venezuela (163), y a Brasil, con 62, en décimo sitio junto a Nicaragua. En radio, elíndice de habitantes por receptor ubica a Brasilen el quinto lugar latinoamericano con 2.5\% y a México en el decimoquinto por 5.1. El mismo índice en televisión es 4.0 para Brasil, el segundo de América Latina, y de 8.7 para México, el decimosegundo. La gran mayoría de los largometrajes latinoamericanos son producidos en los dos países: en 1988 Brasil produjo 73 y México 83 de las 226 películas de los 21 países. El índice de asistencia al cine, por cada mil habitantes, resulta de 730 (undécimolugar)en Brasilyde2730 en México(tercero).Por supuesto, es necesario mucho más análisis y mejores datos para trabajar algunas conclusiones, pero esa tarea es avanzada por otros colegas, por lo que no puede recuperarse aquí.

Lo que puede tomarse como punto de partida es la gran importancia relativa que, en América Latina, tienen Brasil y México en prácticamente todos los órdenes. Sus economías los sitúan en el mundo como países "límite" entre las naciones hegemónicas y las que parecen condenadas irremediablemente a ser dependientes; de ahí su papel definitivo para América Latina en la integración comercial respectiva del Mercosur y el NAFTA; de ahí también la importancia de sus procesos políticos, tanto de estabilización "democrática" como de "modernización" y el protagonismo internacional de sus presidentes, en el caso de Brasil interrumpido por la renuncia de Fernando Collor de Mello a quien, simplificando mucho, se dice que lo hizo presidente la televisión (Lins da Silva, 1991) y lo removió la prensa (Castro, 1993; Torquato, 1993; Moreira, 1993). 
Los afanes de "modernización" y de inserción privilegiada en el nuevo orden global son indudablemente prioridades de las élites gubernamentales y empresariales de Brasil y de México, países muy ricos en recursos y ambiciones. Pero los obstáculos internos que ambos deben superar para avanzar en ese proyecto son enormes y se resumen en una condición: la desigualdad social, manificsta en todas las dimensiones económicas, políticas y culturales de sus estructuras y sistemas, incluyendo por supuesto las educativas y comunicacionales, en cuya intersección ("campo académico de la comunicación") se sitúa el objeto de atención de este artículo.

\section{El campo académico latinoamericano de la comunicación: un segundo contexto}

En los ámbitos de la comunicación social y de su estudio, los problemas, avances y perspectivas brasileñas y mexicanas son inevitablemente influyentes en el resto de América Latina. Si se desglosan elementalmente las cifras latinoamericanas correspondientes al campo académico de la comunicación (enseñanza e investigación), un tercio de cualquiera de ellas corresponderá a Brasil, otro a México y el restante a los otros 19 países del área. El investigador norteamericano Emile McAnany establecía recientemente el siguiente marco, hablando de la cooperación entre América Latina y los Estados Unidos:

Elpapel de la investigación en América Latina yla posibilidad de una mayor colaboración con otros provenientes de fuera de la región no pueden permanecer aislados del entrenamiento y de la estructura académica. Parte de esta situación es cómo los investigadores son entrenados y cómo la investigación es generada en las instituciones académicas y de investigación en la región. Si los datos de Fuentes Navarro (1992) son correctos, para 1990 había cerca de 244 escuelas y departamentos de comunicación en Latinoamérica, con 5000 profesores universitarios y cerca de cien mil estudiantes. El autor resalta en su artículo algunos de los problemas que la disciplina enfrenta en la región: una desarticulación entre las metas profesionales y de investigación en muchas instituciones; una separación entre la investigación teórica y aplicada; una separación entre los compromisos sociales y políticos y la preparación de los estudiantes para que se incorporen a la industria, de la cual muchos académicos desconfían; los problemas muy prácticos de fusionar las metas políticas y de investigación involucradas en el financiamiento externo. 
De alguna manera el escenario de las escuelas de comunicación en América Latina es bastante distinto del de los Estados Unidos, donde la tradición del funcionalismo/positivismo permanece fuerte, si no es que dominante y donde, hasta hace bastante poco, financiamientos provenientes de una variedad de fuentes privadas y públicas le dieron a algunos investigadores presupuestos para hacer la clase de estudios que frecuentemente son obstaculizados cuando la investigación es auto-financiada. Actualmente ha habido una reducción sustancial en el financiamiento público y se ha empezado a desarrollar una tradición en comunicación en estudios críticos para compensar el viejo funcional-positivismo. Al leer la descripción de Fuentes Navarro de los problemas que actualmente están enfrentando los académicos, yo pude identificar bastante bien muchos de ellos (McAnany, 1992: 342-343).

Las cifras citadas en el artículo referido por McAnany no sólo son correctas, sino que quedan cortas, ya que representan una aproximación mínima, dependiente de la incompleta información que se hace llegar a la Federación Latinoamericana de Asociaciones de Facultades de Comunicación Social (FELAFACS), el organismo de coordinación académica más importante del área en el subcontinente. Adoptando el marco comparativo con Estados Unidos como lo plantea McAnany, se facilita la exposición de los sistemas brasileño y mexicano para el estudio de la comunicación, dentro del contexto latinoamericano. De otra manera, los parámetros nacionales aislados pueden impedir la consideración de los procesos y de los proyectos que generan tanto los números como sus interpretaciones.

La apreciación de McAnany parece suponer que los investigadores latinoamericanos de la comunicación son "entrenados" en las universidades como los norteamericanos. Este supuesto requiere la exploración de varios implícitos, que McAnany nodesconoce pero que por razones de exposición no analiza, quedándose en la clásica dicotomía entre las corrientes "crítica" y "administrativa" y su relación con el financiamiento. El primer factor implícito es el carácter casi exclusivamente profesionalizante de las escuelas latinoamericanas de comunicación, de entre las cuales sólo una de cada diez cuenta con programas de maestría y una de cada cien con doctorado. El segundo factor, estructuralmente articulado con el anterior, es que por el crecimiento apresurado del sistema educativo, la formación de los profesores e investigadores no supone, como en los Estados Unidos, el doctorado como requisito para acceder a un puesto académico en las universidades y que quienes cuentan con posgrados en América Latina lo obtuvieron mayoritariamente en otra área disciplinaria o bien en otro país (Estados Unidos o Europa). El tercer factor, también estructuralmente articulado con los anteriores, y claramente de- 
sarrollado por McAnany, apunta al grado de legitimación y de institucionalización alcanzado por la investigación de la comunicación, objeto central de este artículo:

Una de las principales diferencias entre las dos regiones que yo sugeriría radica en la relación existente entre los estudios en comunicación y las ciencias sociales tal como Fuentes Navarro y otros artículos del número de Diá-logos intentan explicar. Una diferencia con los Estados Unidos puede ser la relación tradicional entre la universidad y la gran sociedad civil, el gobierno y las fundaciones y, más recientemente, el sector privado (el cual cada vez más está financiando ciertas clases de investigación universitaria). Ha habido un rol tradicional para las ciencias sociales (y comunicación frecuentemente se incluye como tal) en la vida académica americana y los problemas sociales desde principios de este siglo (...). El nacimiento del campo académico de la comunicación ciertamente conllevó fuertes nexos del financiamiento Rockefeller con Paul Lazarsfeld en el estudio del rol social de la radio en 1934 (...), así como las aplicaciones de la investigación de la comunicación a los diferentes esfuerzos para movilizar y entrenar a las personas durante la segunda guerra mundial (...). Lo que esta tradición ha significado ha sido que la investigación aplicada, a menudo con una agenda social, era parte del desarrollo académico institucional. A pesar de que el financiamiento para este tipo de investigacion se ha visto sustancialmente reducido en años recientes, permanece vigente una fuerte tradición de la investigación social aplicada relacionada con amplias cuestiones de la política pública y aún con perspectivas críticas en las escuelas de comunicación de los Estados Unidos. En la medida que han surgido nuevas tecnologías y han ocurrido cambios en las estructuras de la comunicación y la globalización de las industrias de la comunicación en los Estados Unidos, la investigación universitaria ha tratado de mantenerse al tanto de estos cambios tanto como le fuera posible debido a la importancia teórica y a la relevancia que esto tenía para sus propias políticas.

La razón por la cual se hacen estas observaciones es para sugerir que las tradiciones institucionales en los Estados Unidos han hecho posible no solamente proveer la educación a profesionales que se incorporaran a las industrias de la comunicación, sino también el entrenar a los investigadores que estudiaran pero que no se incorporaran a dichas industrias. Esta investigación varía grandemente en su perspectiva, alguna como investigación administrativa provee a la industria con mejor conocimiento acerca de su funcionamiento, otra como investigación crítica sirve para criticar a la misma industria y proveer las alternativas políticas para un debate. ¿Cómo, pues, pueden estas tradiciones ser una base potencial para la colaboracion? (McAnany, 1992: 343-344)

Nuestro propósito no llega hasta la colaboración, pero dado que el sistema norteamericano de educación superior, de investigación, de formación profesional y de elaboración teórica es el modelo sobre el cual se ha estructurado el campo 
académico de la comunicación en todo el mundo, y considerando que tanto en Brasil como en México hay constantes impulsos de ciertos agentes del campo nacional para tomar como propios los parámetros norteamericanos, creemos pertinente partir del planteamiento (crítico y cercano a América Latina) de McAnany para tratar de documentar los procesos de institucionalización del estudio de la comunicación en los dos mayores países latinoamericanos.

Conceptualmente, como la mayor parte de los investigadores de estos temas, consideramos que la institucionalización de un campo académico comprende dos planos o procesos paralelos, ninguno de los cuales puede ser ignorado y que se desarrollan en estrecha vinculación mutua: por una parte la institucionalización social, que se centra en las relaciones establecidas entre los agentes académicos y la sociedad, y por otra la institucionalización cognoscitiva que consiste en compartir, los académicos de un campo, un enfoque común de los objetivos, metas y métodos específicos de ese campo. En este doble sentido, la institucionalización es la manifestación más "objetiva" de la constitución de un campo académico, cuando las instancias del poder social asignan o reconocen un lugar específico a la producción y reproducción del conocimiento sobre un área determinada e, implícita o explícitamente definen la orientación y el sentido (función social) que el trabajo sobre dicha área en dicho lugar deberá de cumplir para reforzar su legitimidad. Este proceso es entonces inseparable de la profesionalización de los sujetos que, dentro de las instituciones establecidas, habrán de ejercer las prácticas académicas y articula, de maneras más o menos "fuertes", la producción académica con la toma de decisiones en el área, lo cual a su vez contribuye a la legitimación del campo, de las instituciones y de los sujetos que lo componen.

En el caso del estudio de la comunicación en México, hemos encontrado un incipiente y complejo pero claro proceso de legitimación dentro del campo más general de las ciencias sociales mediante la constitución de una sólida "comunidad académica", aún desapercibida (Fuentes, 1991a), aunque los colegas brasileños documentan un estado de avance mucho mayor en su país.

\section{Dos sistemas académicos hipertrofiados}

Como se ha señalado anteriormente, es en Brasil y en México donde se ha concentrado, aunque de maneras distintas, el mayor peso del desarrollo del campo académico de la comunicación en América Latina, especialmente en cuanto a 
programas de formación profesional, que tienen su origen en las escuelas de periodismo fundadas a partir de los años treinta sobre el modelo profesionalizante consagrado en los Estados Unidos por Joseph Pulitzer (Nixon, 1974). En 1950 había doce de estas escuelas en Latinoamérica, de las cuales dos estaban en Brasil y dos en México. En 1960 había 44 y el total pasó a 81 en 1970 y a 163 en 1980 (Nixon, 1981); el patrón de duplicación por década se mantuvo, para llegar a alrededor de 300 en 1990, de los cuales más de cien se localizan en México y más de 70 en Brasil (FELAFACS, 1992).

El estudio más completo y sistemático sobre el sistema de formación de comunicadores en Brasil es el Diagnóstico das Escolas de Comunicação Social 1986-1987, preparado por una comisión de especialistas coordinada por José Marques de Melo y un grupo de trabajo dirigido por Antonio Carlos de Jesús para el Ministerio de Educación (1987). En él analizan la información proporcionada por las 66 escuelas entonces operantes en Brasil. En cuanto a la investigación, en 1990 se publicó un excelente estudio evaluativo elaborado para el Consejo Nacional de Desarrollo Científico y Tecnológico por Sergio Caparelli (1990). Habremos de citar ampliamente ambos documentos en lo subsiguiente. Lamentablemente en México no se cuenta con estudios equivalentes. El diagnóstico más completo sobre la formación de comunicadores fue elaboradoentre 1980 y 1981 por la Coordinación General de la Presidencia de la República, cubriendo entonces 27 instituciones. A pesar de haber quedado inédito, algunos datos fueron rescatados por el CONEICC (1981), Beatriz Solís (1983) y Alberto Rojas (1983). Precisamente en la década en que el crecimiento de las escuelas de comunicación mexicanas fue explosivo, los únicos esfuerzos por mantener actualizada la información, realizados por el CONEICC, son notablemente insuficientes y no han tenido ningún carácter evaluativo.

Esta es una primera y notable diferencia entre ambos países, que se remite al papel que han desempeñado los respectivos gobiernos en la regulación de los sistemas de educación superior. En Brasil, las carreras de comunicación deben seguir un "curriculum mínimo" general, establecido por el Consejo Federal de Educación, cuyas resoluciones permiten que cada universidad complete su "curriculum pleno" y ofrezca una o varias de las "habilitaciones" aprobadas: Periodismo, Producción Editorial, Radiodifusión, Publicidad y Propaganda, Relaciones Públicas y Cine. Caparelli sintetiza así la historia curricular brasileña de las carreras de comunicación: 
Los cursos de graduación vivieron durante muchotiempo la búsqueda de una identidad, parcialmente reflejada en la estructuración de su currícula. Esos currícula surgieron en contextos históricos, políticos, económicos y sociales determinados. El primero, de 1962, fue estructurado dentro de una perspectiva liberal: tenía apenas doce materias obligatoriasy recomendaba la necesidad de otras, dejadas abiertas, a partir del supuesto de que la especificidad del periodismo está en su generalidad. Fil segundo, de 1968, resulto de un control autoritario sobre la universidad y los inicios de una reforma universitaria que buscaba la cualificación de recursos humanos para hacer avanzar el modelo económico. Ese curriculum estuvo caracterizado en consecuencia por una excesiva tecnificación de las carreras. El tercer curriculum [1977] reconoció esto y propuso que se pasara de lo que califico como la fase científico-técnica a la fase crítico-reflexiva. (...)

En los años 80 las carreras se están reestructurando a partir de la aprobación del curriculum mínimo a través de la aprobación de la resolución 02/84 del 6 de octubre de 1983. Este curriculum mínimo provoco algunos cambios en la estructura curricular y, desde su aprobación por el Consejo Federal de Educación hasta 1986 las escuelas todavía trabajaban en su adaptación.

Para las escuelas de comunicación, el acuerdo 480/88 fue importante por establecer por primera vez exigencias de una infraestructura para el funcionamientode las carreras, en términos de laboratorios y equipos, así como de una infraestructura pedagogica. Para los establecimientos públicos fue la oportunidad de que las escuelas solicitaran el cumplimiento de las exigencias que provenían del propio Ministerio encargado de proveera las escuelas públicas federales de los recursosindispensables para su ejecución. Los establecimientos privados se vieron en la contingencia de ofrecer mejores condiciones de funcionamiento. (Caparelli, 1990:6-8).

En México, casi todas las instituciones tienen libertad, legal o de facto, para establecer su curriculum, por lo que no es sorprendente que, desde el nombre mismo de la carrera, se encuentren más de 25 versiones distintas del título de licenciatura otorgado en ellas. Varias de las "habilitaciones" brasileñas cuentan con un ejercicio profesional oficialmente reglamentado, lo cual no sucede en ningún caso en México.

Aunque no hay datos disponibles sobre la distribución por carreras o áreas disciplinarias de los aproximadamente millón y medio de estudiantes brasileños de educación superior, en 1986 se registraron 25,425 alumnos matriculados en "bacharelado" en 66 escuelas de comunicación, la gran mayoría de los cuales cursaban Periodismo, Publicidad y Propaganda y Relaciones Públicas. (MEC, 1987) Hay que recordar que, a diferencia de casi todos los demás países latinoamericanos, en Brasil las carreras de comunicación conservan como base el proyecto "especi- 
ficante" y no el "generalizante" que ha sido, desde los años sesenta, objeto de debate en cuanto al perfil profesional y curricular de las escuelas. No obstante, la adscripción institucional de las carreras de comunicación en la estructura universitaria por áreas disciplinarias es en Brasil tan heterogénea como en los demás países del área. Dos tercios de las 66 escuelas brasileñas están integradas a facultades, escuelas o departamentos universitarios y 22 , todas ellas privadas, tienen una adscripción institucional más o menos "aislada", incluyendo dos establecimientos que sólo ofrecen carreras de comunicación, mientras que en el otro extremo, la Escola de Comunicaçoes e Artes de la Universidade de São Paulo, sin duda la más importante del país, incluye once carreras o "habilitaciones" profesionales: Biblioteconomía y Documentación, Cine, Periodismo, Radio y Televisión, Relaciones Públicas, Teatro, Producción Editorial, Publicidad y Propaganda, Turismo, Artes Plásticas y Música (Lopes et al, 1992). Esta adscripción de la Comunicación junto a las Artes es casi única en América Latina, pues por logeneral las carreras de comunicación son, o bien "independientes", o bien parte de facultades o departamentos de Ciencias Sociales. Lo que es claro es que en ninguna parte de esta ubicación estructural, aspecto fundamental de su institucionalización, está definitivamente resuelta.

Por su parte, en México predomina el modelo "generalizante" de formación profesional, bajo la denominación de "licenciatura en ciencias de la comunicación" o similares, en muchos casos con "menciones" de especialización en el título, pero bajoun curriculum común. Según datos de la Asociación Nacionalde Universidades e Instituciones de Educación Superior (ANUIEs), la carrera alcanzó en 1991 el décimo lugar entre las más pobladas en el país. De los más de 900 mil estudiantes de licenciatura, las carreras de Contador Público y Derecho absorbieron cada una más de 100 mil alumnos; Administración alcanzó casi esa suma; la carrera de Médico Cirujano, a pesar de haber disminuido su matrícula con respecto a 1985 , se mantuvo en cuarto lugar con más de 55 mil estudiantes, seguida de Ingeniería Industrial, Eléctrica-Electrónica y Mecánica Eléctrica, con entre 40 mil y 50 mil alumnos cada una; tanto Arquitectura como Ingeniería Civil rebasaron los $45 \mathrm{mil}$ estudiantes y Ciencias de la Comunicación se convirtió por primera vez en una de las diez carreras universitarias más demandadas, con 26,393 estudiantes inscritos (Gago, 1992:30). El CONEICC considera para el mismo 1991 una cifra de estudiantes ligeramente superior: 27,319 estudiantes, inscritos en 70 instituciones (CONEICC,1991). 
La comparación de cifras es extremadamente difícil en América Latina por la escasez y poca confiabilidad y disponibilidad de fuentes estadísticas. De cualquier manera, si calculamos conservadoramente que hay alrededor de 30 mil estudiantes de comunicación en cada uno de los dos países de referencia, y aproximadamente dos mil profesores en cada uno de ellos, y que estos números tienden a crecer aunque más moderadamente que en los ochenta, podemos afirmar que la institucionalización del campo académico de la comunicación, al menos en su aspecto cuantitativo y desde el punto de vista de los programas de formación profesional, es sobradamente relevante en estos dos países y que vale la pena analizar las condiciones de su consolidación académica, pues la dinámica de crecimiento y la magnitud alcanzada son motivo más de preocupación que de satisfacción para la mayor parte de sus testigos y protagonistas tanto en México como en Brasil.

En todo caso, las cuestiones de la pertinencia, la calidad y la eficiencia de la formación profesional de los comunicadores son objeto de constante debate al menos desde los años sesenta, tanto al interior de la "comunidad académica" de ambos países como ante las instancias del poder político y económico, los empleadores y los propios estudiantes, pues indudablemente la más fuerte, y en muchos casos casi única, justificación social de la existencia y desarrollo del campo académico ha sido su referencia (en muchos sentidos dependencia) al sistema de medios masivos o industria cultural que tanto en Brasil como en México ha tenido especial importancia para el "desarrollo nacional" y ha alcanzado un nivel de avance (económico, político y cultural) competititvo en ciertas áreas a nivel global.

Sin entrar aquí a describir o a participar en el debate actual sobre la orientación y la calidad de las escuelas de comunicación, que se ha concentrado para América Latina en la revista Diá-logos, de FELAFACs (Neira, 1990; Sánchez García, 1991; López Veneroni, 1991; Caletti, 1991; Fuentes 1991b, 19924, Quiroz, 1991, Marques de Melo, 1991b; Andión, 1991; Fadul, 1992; Martín Barbero, 1990a, 1990b, 1992) puede postularse una hipertrofia, especialmente notable en Brasil y México, del sistema académico latinoamericano para el estudio de la comunicación, que ha obstaculizado (cuando no impedido) el desarrollo profesional, teórico, educativo y social general del área y una polarización institucional cada vez mayor: especialmente, otra vez, en Brasil y en México, se incrementa la "distancia académica" entre unas pocas instituciones universitarias que cuentan con los recursos y los proyectos necesarios para aspirar a la "excelencia" y una gran mayoría de estable- 
cimientos cuyo propósito es casi únicamente comercial o que son incapaces de participar siquiera en los debates sobre el desarrollo académico del campo.

En Brasil, como ya se ha dicho, la intervención gubernamental a través de diversas instancias del Ministerio de Educación frenó el crecimiento anárquico de las escuelas de comunicación y diversas organizaciones y líderes académicos han hecho pesar su opinión en la toma de decisiones regulatorias oficiales. En México eso no ha sucedido, ni parece posible a corto plazo. No obstante, las políticas recientemente impulsadas por la Secretaría de Educación Pública para la educación superior, especialmente por las vías financieras, tenderán sin duda a exacerbar la polarización, aunque no detendrán el crecimiento anárquico, al menos a corto plazo.

\section{La investigación y el posgrado como instancias de legitimación académica del campo}

Uno de los aspectos indispensables para la legitimación de un campo académico es el desarrollo de la investigación y la profesionalización avanzada que, en relación con ella, promueven los posgrados en el área de conocimiento propia. La investigación y los posgrados son así indicadores especialmente importantes del grado de institucionalización de un campo académico, pues en lo social implican el otorgamiento de recursos financieros y de reconocimiento oficial, escasos por definición, y en lo cognoscitivo una estructura teórico-metodológica e ideológica mínima compartida por los agentes del campo. Los sistemas universitarios para el estudio de la comunicación de Brasil y de México, a pesar de la hipertrofia indicada en cuanto a la formación de profesionales, han desarrollado desde los años setenta espacios relativamente importantes para la investigación y el posgrado, prácticamente como casos únicos en América Latina. Según Brunner, esto se extiende a los posgrados en general y a la investigación científica (1990: 133-160).

Para apreciar mejor las dimensiones del desarrollo de la investigación y el posgrado en comunicación en ambos países, partiremos de las plantas docentes empleadas en los sistemas respectivos, ya que obviamente son los académicos (profesionales de la enseñanza-investigación universitaria) los sujetos responsables de las actividades en proceso de institucionalización. Como lo han señaladodiversos estudios sociológicos sobre estos agentes, hay una relación muy estrecha entre el número de profesores "de carrera" (contratación de medio tiempo, completo o 
exclusivo) y el desarrollo de la investigación en las instituciones. De esta manera, con profesores contratados "por hora", puede sostenerse un programa de formación profesional pero difícilmente un posgrado o un programa de investigación de calidad. Los datos agregados de las plantas docentes de las escuelas de comunicación brasileña y mexicana, distinguiendo instituciones públicas y privadas en ambos casos, indican las siguientes proporciones:

Tabla 3. Planta docente en comunicación en Brasil y en México

\begin{tabular}{c|cc|cc}
\hline $\begin{array}{c}\text { Régimen de } \\
\text { contratación } \\
\text { académica }\end{array}$ & $\begin{array}{c}\text { Instituciones } \\
\text { públicas en } \\
\text { Brasil (1986) }\end{array}$ & $\begin{array}{c}\text { Instituciones } \\
\text { privadas en } \\
\text { Brasil (1986) }\end{array}$ & $\begin{array}{c}\text { Instituciones } \\
\text { públicas en } \\
\text { México (1991) }\end{array}$ & $\begin{array}{c}\text { Instituciones } \\
\text { privadas en } \\
\text { México (1991) }\end{array}$ \\
\hline Por horas & $68(9 \%)$ & $812(83 \%)$ & $606(70 \%)$ & $1,224(78 \%)$ \\
\hline De carrera & $652(91 \%)$ & $165(17 \%)$ & $264(30 \%)$ & $334(22 \%)$ \\
\hline
\end{tabular}

Como se ve en la tabla, la mayor parte del personal docente en comunicación tanto en Brasil como en México, está contratado por horas en las escuelas: el 52\% de los profesores brasileños y el $75 \%$ de los mexicanos. Esa diferencia de proporciones entre los dos países, más allá de las fechas y la confiabilidad de las cifras, indica un grado bastante mayor de profesionalización institucionalizada en el "mercado" académico brasileño que en el mexicano pues, mientras en Brasil hay 817 profesores de carrera, en Mćxico se cuentan sólo 598, y los primeros representan el 48\% de la planta docente de su país, mientras que los segundos sólo el $25 \%$ de la propia. La distinción por instituciones públicas y privadas nos indica otra diferencia notable: aunque en ambos países hay más escuelas de comunicación públicas que privadas ( 37 por 29 en Brasil y 70 por 22 en México) en Brasil la proporción de estudiantes de $73 \%$ en las privadas y $27 \%$ en las públicas, mientras que en México es de $45 \%$ en las privadas y $55 \%$ en las públicas (Caparelli, 1990; CONEICC, 1991) y esto puede significar, de entrada, diferencias importantes entre un país y otro.

Primero, parece implicarse que las instituciones públicas brasileñas tienden a ser muy selectivas y que la mayor parte de los estudiantes que rechazan son los que se inscriben en muchas de las escuelas privadas, aunque entre estas últimas hay varias de gran prestigio, también muy selectivas. Las instituciones públicas mexicanas, por su parte, tienden a ser masivas (poco o nada selectivas) y la demanda de los estudiantes se distribuye casi por igual entre pocos grandes establecimientos 
públicos y muchos pequeños privados. Aunque aquí habría que hacer un análisis más fino, por ejemplo regional, en cada país, pues la concentración de escuelas y estudiantes en los grandes centros urbanos (Sao Paulo y Río de Janeiro, la Zona Metropolitana de la ciudad de México) es muy considerable en ambos casos. En síntesis, podría asegurarse que hay relativamente buenas condiciones para el desarrollo académico en más instituciones brasileñas que mexicanas, tanto públicas como privadas.

Por otra parte, el control homogéneo que el Ministerio de Educación brasileño ejerce sobre todas las escuelas de comunicación, independientemente de su régimen jurídico (instituciones federales, estatales, municipales, confesionales o particulares), significa también una tendencia a la aplicación de criterios homogéneos para la asignación de apoyos (becas, financiamiento de proyectos, etcétera) y la evaluación, aunque evidentemente haya disparidades de hecho. En México la tendencia oficial es, hasta hace poco, concentrar los apoyos (y las restricciones) en las instituciones públicas, también con disparidades de hecho, y dejar depender de sus propios medios a las instituciones privadas. $\mathrm{Al}$ mismo tiempo, la remuneración de los profesores brasileños tiende a ser mucho mejor en las universidades públicas que en las privadas, mientras que en México se tiende a la situación opuesta.

Decualquier manera, es claro que en ambos países las posibilidades de desarrollo del campo a través de la investigación y el posgrado están limitadas a unas cuantas instituciones, públicasy privadas, aunque sean un poco más en Brasil que en México, que destacan como "centros concentradores" de recursos acadćmicos en el campo, cada vez más "distantes" y de alguna manera "dominantes" de la mayor parte de las escuelas del respectivo país. Por sus recursos docentes, resaltan en Brasil la Universidad de São Paulo (más de 200 profesores de tiempo completo) y en México la Universidad Autónoma Metropolitana-Xochimilco (más de 75). A partir de aquí, es necesario subrayar que hay una relación doble entre plantas docentes e investigación, por un lado, y entre ésta y el carácter predominantemente académico de la producción de conocimiento, al menos la que puede documentarse como pública, tanto en Brasil como en México:

Un inventario reciente que realizamos [en Brasil] revelo que más del $80 \%$ de los investigadores de la comunicación son profesores universitarios y que en las universidades se realiza el $90 \%$ de la producción científica en comunicación, lo cual equivale 
a decir que esa producción es de carácter predominantemente académico (Lopes, 1991: 180).

Cifras y proporciones semejantes se encuentran en la documentación mexicana de la investigación de la comunicación:

Por la obvia razón de que sus resultados no se publican, es muy escaso el número de investigaciones "comerciales" o realizadas privadamente para un cliente determinado que se incluyeron. (...) Predominan, en consecuencia, los productos de la investigación "académica", realizada principalmente en las universidades, sin fines de lucro y menor vinculación con las decisiones que conforman el panorama comunicacional mexicano (Fuentes, 1988: 14-15).

Hasta 1988, operaban en Brasil seis programas de maestría y los únicos tres de doctorado en Comunicación existentes en América Latina. De esa fecha a hoy ha habido algunos cambios y adiciones, difíciles todavía de documentar, por lo que nos limitaremos a los datos anteriores. Las tres universidades con los dos niveles de posgrado son la de São Paulo (pública), cuya maestría inició en 1972 y el doctorado en 1982; la Federal de Río de Janeiro (pública), con maestría desde 1972 y doctorado desde 1983; y la Católica de São Paulo (privada), cuya maestría data de 1978 y el doctorado de 1981. Ofrecen maestrías, además, la Universidad de Brasilia (pública), desde 1974; el Instituto Metodista de Enseñanza Superior (privada), desde 1978; y la Universidad de Campinas (pública), a partir de 1986. Cada uno de los programas tiene su propio enfoque y especialidades de investigación, y diversa productividad por años, como puede verse en la tabla No. 4.

La evaluación cualitativa de estos programas de posgrado avala su consolidación académica, y las instancias oficiales son muy exigentes para aprobar la apertura de nuevos programas. Un comentario de Caparelli es, entre otros, muy ilustrativo:

Los diversos programas de posgrado (...) resaltan la importancia de la diversidad de las experiencias que, orientadas por una pluralidad de perspectivas científicas, no se oponen sino que se complementan, y permiten encarar el sampo teórico de la Comunicación como una área científica fuertemente relacionada con las ciencias sociales y humanas. (...) De hecho existe la interdisciplinariedad necesaria en el abordaje de la comunicación y, al mismo tiempo, una paulatina conquista de una identidad en tanto área autónoma de las ciencias humanas. (...) A pesar de ser unárea nueva, sus programas de posgrado ya forman un número casi igual de maestros y de doctores que los de las áreas próximas. Para esa comparación, tomamos la trayectoria cuantitativa de progra- 
mas de ciencias sociales y humanas, como Antropología, Sociología, Ciencia Política, Economía e Historia. Algunos de ellos bastante académicos, otros caracterizados por su aplicabilidad. El periodo analizadoes de 1981 a 1986. De esos cinco cursos, el número de formados en Comunicación es muy cercano o hasta levemente superior a los de Antropología y Ciencia Política, siendo inferior a Sociología, Economra e Historia, tres áreas ya consolidadas hace bastante tiempo. Tanto los programas de Antropología como los de Ciencia Política antecedieron a los de Comunicación en las universidades brasilefias (Caparelli, 1990: 19-20).

Tabla 4. Posgrados en comunicación en Brasil hasta 1988

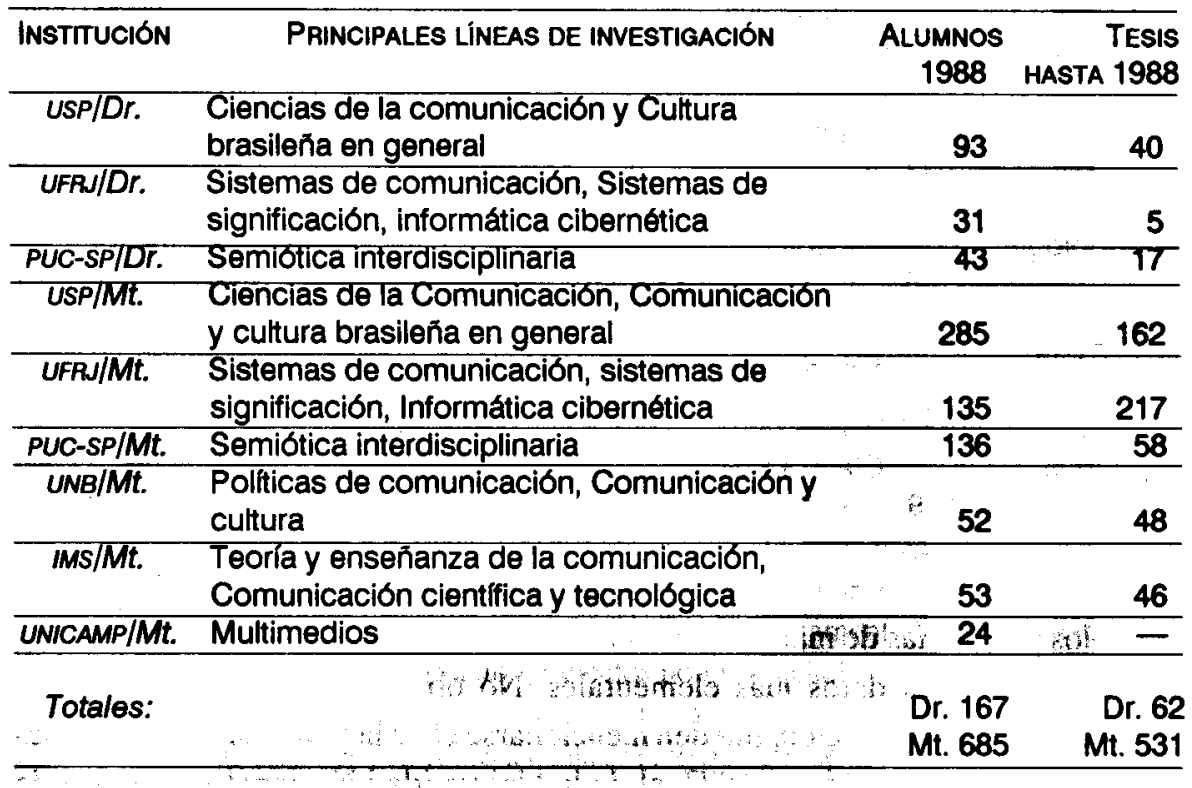

Tanto en Brasil como en México, no obstante, la mayor parte de los profesores e investigadores con posgrado lo han estudiado en Europa o Estados Unidos. En Brasil, a pesar del crecimiento de los posgrados nacionales en Comunicación, tanto en relación con otras disciplinas (lo cual indica un grado creciente de legitimación), como en cuanto a la propórción de los académicos que cuentan con esa calificación (índice a su vez de profesionalización), se sigue buscando y apoyando oficialmente a través de becas la posgraduación en el extranjero. Las maestrías mexicanas han contribuido en mucho menor proporción a la formación de la planta académica nacional, además de que quienes, por la restricción en las becas al extranjero o por 
su propia elección, cursan doctorados en el país, lo hacen en Ciencias Sociales, Sociología, Antropología, etc., al no haber la opción de programas en Comunicación. En razón de lo anterior, no puede compararse el número de maestros y doctores formados en distintas disciplinas en México con Brasil, aunque una revisión rápida de los miembros del Sistema Nacional de Investigadores por áreas de las Ciencias Sociales, donde la categoría correspondiente se llama "Información", da una idea de que ni la legitimación ni la profesionalización de la investigación de la comunicación en México alcanzan los niveles de Brasil.

Tabla 5. Miembros del Sistema Nacional de Investigadores Área de Ciencias Sociales, por disciplina, México, 1990

\begin{tabular}{|c|c|c|c|c|c|c|c|}
\hline Historia & & Literat., Filología & & Derechoy & & Arquitectura & \\
\hline & 211 & y Bellas Artes & 85 & Jurisprudencia & 51 & & 15 \\
\hline Antropologia & & Filosoffa & & Psicologla & & Administración & \\
\hline & 138 & & 58 & & 50 & & 11 \\
\hline Sociología & & Educación & & Lingülstica & & Biblioteconomía & \\
\hline & 137 & & 55 & & 39 & y Archivonomía & 8 \\
\hline Economia & & Ciencia Política & & Demografía & & Información & \\
\hline & 119 & y Admón. & 53 & & 30 & & 7 \\
\hline
\end{tabular}

Sobre los programas de maestría en Comunicación existentes en México es difícil incluso enlistar los datos más elementales. No obstante, entre los programas orientados a la investigación, pueden mencionarse el de la Universidad Iberoamericana (privada), fundado en 1977; el de la Universidad Nacional Autónoma de México (pública), establecido en 1979; el del rTESo (privada), creado en 1985; y, con propósitos más profesionalizantes que de investigación, las maestrías en Publicidadyen Comunicación Institucional del Centro Avanzadode Comunicación (privado), establecidas en 1990. De las tres primeras en conjunto, puede contarse un total de entre 80 y 100 tesis de grado.

En cuanto a los productos de la investigación sistematizados por el PORT-COM de INIERCOM en Brasil y el Centro de Documentación de CONEICC en México, se encuentran características similares en Brasil y en México, a pesar de la diversidad de condiciones. Desde el punto de vista de las temáticas, Lopes resume las tendencias brasileñas encontradas en 1995 textos: 
En primer lugar, los medios de comunicación masiva (prensa, cine, televisión, propaganda, teatro y radio) aparecen como objeto del $42.3 \%$ de las investigaciones. Así mismo, la temática de lo masivo (medios de comunicación y cultura de masas) es una constante en casi el $50 \%$ de ellas. En segundo lugar viene la temática de lo popular (comunicación y cultura popular, alternativa, recepción), con el $10.3 \%$ y en tercero los estudios sobre política (comunicación política, políticas culturales y de comunicación, propaganda) con 9.5\%. En cuarto lugar aparece la temática del lenguaje (comunicación lingǘrstica, semiologra, literatura) con un $7.5 \%$ y en quinto la temática tecnológica (comunicación rural, difusión de innovaciones, nuevas tecnologías de comunicación) con 7.0\%. Por otra parte, las áreas de teoría e investigación, fundamentales para el desarrollo de la reflexión básica y de la investigación de base en el campo de la comunicación, revelan una producción bajísima (...) En contraste con esa posición marginal ocupada por la investigación teorica y metodologica, es posible identificar una preocupación central por la temática profesional en las investigaciones registradas (Lopes, 1991: 172).

En el correspondiente análisis de la investigación mexicana se encuentran algunas similitudes y ciertas diferencias, aunque la muestra documental es en este caso de 877 documentos y el sistema de categorías empleado, distinto, además de la cobertura temporal, que en Brasil llega a 1988 y en México a 1986:

No es sorprendente que dos de cada tres estudios $(63.1 \%)$ se refieran a los medios, dado que el ámbito masivo de la comunicación es el que ha concentrado mayoritariamente los esfuerzos de investigación no sólo en México sino en todas partes. Más de un tercio de los documentos (34.9\%) vinculan explícitamente la investigación con la planificación y la administración, aspectos no muy alejados de las políticas de comunicación (15.1\%) y de los análisis de la industria (12.9\%). Llama la atención el escaso número de estudios dedicados a analizar la tecnologia de las comunicaciones (3.5\%), que a pesar de ser uno de los aspectos fundamentales del desarrollo de los medios masivos, ha sido dejado de lado... (Fuentes y Sánchez, 1989: 22).

Sise entiende elénfasis "profesional" mencionadopor Lopes en oposición al énfasis "epistemológico" que ella quisiera ver mejor reflejado en la investigación brasileña (Lopes, 1990), la situación es similar a la mexicana, donde también la producción teórica y metodológica es escasa, aunque habría que realizar un análisis más fino para homologar categorías de clasificación y matizar la coincidencia, ya que la proporción de tesis de posgrado en la documentación brasileña es muchísimo mayor que en la muestra mexicana (donde no se consideraron como tales), y es en 
esos trabajos precisamente donde se esperaría encontrar prioritariamente los aportes teóricos y metodólogicos. Sobre este aspecto señala Caparelli:

Estamos conscientes de las dificultades para identificar la dimensión metodológica del trabajo cientifico, más aún en el campo de la Comunicación donde el abordaje multidisciplinar es intrínseco al propio objeto de estudio. No obstante esas dificultades, o será por ellas, se hace urgente iniciar la divulgación del trabajo metodológico en Comunicación, enfrentando todos los riesgos que conlleva caracterizarlo. (...) La investigación de campo, teórica y documental, casi igualmente citadas, caracterizan genéricamente la naturaleza de los estudios de Comunicación. Los investigadores mostraron preferencia por el estudio empírico, teórico y textual de su objeto (Caparelli, 1990: 35-36).

Esta preocupación, y dificultades equivalentes, por el análisis metodológico de las investigaciones se encuentra presente también en México y los resultados preliminares podrían ser similares, aunque los analistas sean quizá más severos:

Además de las principales tendencias que el análisis de esta muestra permite detectar (...) hemos realizado (...) una clasificación de los documentos en razón de su contenido empirico y por la presencia (o ausencia) en ellos de alguna forma de trabajo de campo. Por "contenido empírico" entendemos, en el sentido más amplio, lo que va más allá del "ensayo informado" o de la teorización libre y pura y que representa un esfuerzo organizado de recolección/producción de datos. Así, incluimos los estudios históricos y/o estructurales, los análisis de contenido tanto cuantitativos como cualitativos, las investigaciones por encuestas, los disenos experimentales y la investigación-acción. (...) Resultó que, del total de 877 documentos, sólo 336 (37.9\%) tuvieron contenido empírico en el sentido señalado anteriormente, sin que esto signifique que el resto de los trabajos no usara algún tipo de información factual. Pero puede inferirse que muy probablemente menos de dos quintas partes de los documentos son producto de proyectos formales de investigación (Fuentes y Sánchez, 1989: 15).

La reserva indicada sobre la "severidad" de los analistas proviene de la consideración que de la muestra estudiada estaban excluidas las tesis (de licenciatura o posgrado), sobre las cuales valdría mucho la pena, como en Brasil, emprender un análisis metodológico dado que, por definición en el caso de las tesis de maestría, son productos de proyectos formales de investigación. Esta anotación, sin embargo, sólo quiere enfatizar la "debilidad científica" que sufre la investigación de la comunicación tanto en Brasil como en México, sobre lo cual opina Caparelli: 
La gran dispersión que se nota en los referenciales teóricos, y que nodebe ser confundida con diversidad teórica, parece indicar la persistencia de una situación ya diagnosticada en los primeros balancessobre el estadode la investigaciónen Comunicación en América Latina surgidos al inicio de la década de los ochenta. Ahí se señalaba la fragilidad del trabajo teórico en las investigaciones y la ausencia de un marco conceptual común, como también se indicaba el predominio de las investigaciones descriptivas sobre las interpretativas (Caparelli, 1990: 36-37).

El trabajo de recuperación y síntesis de tales diagnósticos (Marques de Melo, 1991a; Fuentes, 1992b), y de propuestas de desarrollo en el plano metodológico (Lopes, 1990; Sánchez Ruiz, 1992) de la investigación de la comunicación, han sido avanzados tanto en Brasil como en México en los años más recientes y tienen mucho camino aún por recorrer. Por lo pronto, cabe retomar como conclusión de esta sección una cita más de Caparelli, válida tanto para Brasil como para México:

Como se ha visto, las investigaciones en Comunicación se concentran en aquellas instituciones en que los esfuerzos resultaron en una agregación de masa crítica que sirvio de base a los cursos de posgrado. Parece haber una sinergia entre las actividades de maestría y de doctorado y el desarrollo de investigaciones científicas. Los docentes o investigadores más competentes pertenecen a los programas de posgrado, siendo raros los núcleos que sobreviven fuera de ellos. Cuando eso sucede, se trata más de esfuerzos aislados que de una agregación de competencia y recursos que busque objetivos científicos. Son esos cursos de posgrado los que han adoptado programas de investigación, con recursos humanos calificados que actúan también en la docencia, construyendo un espacio académico común (Caparelli, 1990: 37).

\section{Los organismos académicos como agencias de institucionalización}

No cabe duda de que la legitimidad, científica y social, de un campo especializado de estudio depende en gran medida de las formas de su organización tanto dentro de las instituciones educativas comoentre los individuos y los grupos que lo adoptan comosuyo, en espacios académicostransinstitucionales. Por una parte, es inherente al desarrollo científico la formación de "comunidades", redes y "colegios invisibles" no sólo transinstitucionales sino transnacionales; por otra, las publicaciones especializadas y los congresos son los mecanismos esenciales de comunicación entre los practicantes de una disciplina, sobre los cuales se basan en gran medida tanto 
los procesos de profesionalización de los investigadores y su incorporación al reconocimiento como miembro pleno de una "comunidad científica", como los procesos de legitimación del campo y de otorgamiento social de autoridad intelectual a un conjunto de investigadores y a cada uno en particular por el hecho de formar parte del conjunto. En el núcleo de entrecruzamiento de estos planos, el papel de las asociaciones $u$ organizaciones académicas es central para la institucionalización de un campo de estudio.

Este es quizá el aspecto en que con más claridad se manifiesten los diferentes procesos, y sus diferentes etapas de consolidación en Brasil y México, de la institucionalización del campo académico de la comunicación. Mientras en Brasil la organización principal de los investigadores (INTERCOM) tiene mucha mayor "fuerza" y autoridad sobre el campo que la organización dedicada a la enseñanza (ABECOM), en México sucede aún lo contrario: la asociación de investigadores (AMIC) es relativamente mucho más débil que la de escuelas (CONEICC). Para completar este primer acercamiento comparativo entre los campos académicos de la comunicación en México y Brasil, conviene describir a grandes rasgos a estas organizaciones. Para el caso de Brasil, la mejor síntesis histórica la proporciona nuevamente Caparelli:

La primera sociedad científica brasileña de carácter nacional fue la Asociación Brasileña de Enseñanza e Investigación de la Comunicación (ABEPEC), fundada en los años 60. Desde su inicio en 1972 hasta su extinción formal en 1985, sirvio de espacio de debate sobre la comunicación en el país, con sus congresos, coloquios, simposia, y con la publicación de una revista de comunicación. Extinta por problemas de organización, su lugar fue ocupado entonces por la INTERCOM, fundada en 1977. Esa sociedad busc 6 articular a los investigadores brasileños del área de la comunicación. (...) Además de los ciclos anuales de estudios, simposia, seminarios, coloquios y cursos, INTERCOM mantiene un programa de publicaciones constituido por libros, revistas, cuadernos, antologías, obras de referencia y monografías diversas. De 1982 a 1988 publico diez libros, ocho cuadernos, diversas antologías, además de crear el Centro de Documentación de la Comunicación en los Países de Lengua Portuguesa (PORT-COM) para inventariar y divulgar obras relacionadas con la comunicación en los países lusoparlantes. En la segunda mitad de los años ochenta muestra también los primeros resultados de la búsqueda de una cooperación internacional por medio de sociedades científicas, con el inicio de un proyecto de estudio comparado de comunicación entre Brasil y México (Caparelli, 1990: 41-42). 
Habría que anotar que la INTERCOM, simultáneamente al proyecto de referencia con México, estableció similares colaboraciones con España, Francia e Italia, mientras que el coNEICC en México, después de aceptar la propuesta brasileña, apenas cinco años después estudia la posibilidad de establecer proyectos similares con España y Argentina. La historia y el tamaño de INTERCOM, además de su intensa dinámica de trabajo que si bien comenzó en Sao Paulo se ha extendido poco a poco a todo el país, ha generadoya también varias investigaciones profundas sobre sí misma, la más interesante de las cuales quizá sea la de J. S. Faro, titulada significativamente La Universidad Fuera de Sí: INTERCOM y la organización de los estudios de comunicación en Brasil. (1992), en cuyo prefacio se resume el objetivo:

En diciembre de 1977, cuando ya eran visibles las señales de declinación del periodo más largo de autoritarismo vivido por el Brasil, un grupo de profesores fundó en Sao Paulo la Sociedad Brasileña de Estudios Interdisciplinarios de Comunicación - INTERсом- La iniciativa, recubierta de intenciones generosas, traía en su esencia la pretension de ofrecer a la comunidad académica un espacio alternativo para el debate y para el avance de la ciencia, en contrapunto con la insuficiencia de la vida universitaria, marcada por la inhibición y por la fragmentación de sus actividades. Quince ańos despues la INTERCOM está inscrita y consolidada en el proceso de organización de los estudios de comunicación. Más que eso, sus experiencias contribuyeron para que esos estudios adquirieran la densidad correspondiente a las indagaciones provocadas por la extraordinaria complejidad de los fenómenos comunicacionales en la sociedad contemporánea (Faro, 1992: 7).

El estudio de Faro, así introducido, describe en detalle la trayectoria de la INTERCOM, pero sólo después de analizar por una parte, el periodo final de los veinte años de dictadura militar y gobiernos autoritarios que sufrió Brasil, con la consecuente "rearticulación de la sociedad civil" durante el Gobierno Geisel, y por otra, la crisis de la universidad y las condiciones cambiantes de la relación entre los intelectuales y el Estado, como contexto histórico en que se funda la asociación. En México, nadie ha escrito la historia de la AMIC o el CONEICC, a pesar de haber sido fundados respectivamente en 1979 y 1976, y mucho menos analizado las relaciones entre el contexto sociopolítico mexicano, las universidades, los intelectuales y las organizaciones académicas de la comunicación. Difícilmente se guarda algún registro de su papel en la institucionalización del campo, más allá de los muy insuficientes intentos (Fuentes, 1988; 1991) de este autor. Para el propio campo y para sus "habitantes", sobre todo para los de más reciente ingreso a las 
tareas de investigación, conocer críticamente esa historia y participar en su consolidación, parece ser una urgencia que vale la pena atender.

\section{Referencias bibliográficas}

ANDIón Mauricio (1991): "La formación de profesionales en comunicación". Diá-logos de la Comunicación Núm. 31, pp.55-63. Lima:FEL.AFACS.

BRUNNER José Joaquín (1990): Educación Superior en América Latina, cambios y desafios. Chile: Fondo de Cultura Económica.

CALETTI Sergio (1991): "Profesiones, historia y taxonomías: algunas discriminaciones necesarias". Diá-logos de la Comunicación Núm. 31, pp. 25-36. Lima: FELAFACS.

CAPARElli Sergio (1990): “A pesquisa em Comunicação no Brasil: avaliaçao e perspectivas-CNPq". INTERCOM-Revista Brasileira de Comunicaçāo Núm. 62/63, pp.5-45. São Paulo:INTERCOM.

CASTRO Rondón de (1993): “¿Renacimiento espiritual en los medios brasileños?”. Chasqui Núm. 44, pp. 8-9. Quito: CIESPAL.

CoNEICC (1981): Diagnóstico sobre la enseñanza de la comunicación en México. México: Comité de Asuntos Académicos.

CONEICC (1991): Catálogo de Instituciones de Enseñanza Superior en Ciencias de la Comunicación: licenciaturas, posgrados y centros de investigación 1991-1992. México.

EnCyClopaedia BRTANNICA (1990): Britannica World Data Annual. Chicago.

FADUL Anamaría (1992): "Las ciencias sociales en la enseñanza y la investigación de la comunicación”. Diá-logos de la Comunicación Núm. 32, pp.4-7. Lima: FELAFACS. 
FELAFAcs (1992): Directorio de Facultades y Escuelas de Comunicación en América Latina. Santafé de Bogotá.

FueNTES NAVARRo Raúl (1988): La Investigación de Comunicación en México. Sistematización Documental 1956-1986. México: Ediciones de Comunicación.

(1991a): La Comunidad Desapercibida. Investigación e Investigadores de la Comunicación en México. Guadalajara: ITESo/CONEICC.

(1991b): "Prácticas profesionales y utopía universitaria: notas pararrepensar el modelo de comunicador" Diá-logos de la Comunicación Núm. 31, pp. 37-42. Lima: FELAFACS.

(1992a): "El estudio de la comunicación desde una perspectiva sociocultural en América Latina". Diá-logos de la Comunicación Núm. 32, pp. 16-27. Lima: FELAFACS.

(1992b): Un campo cargado de futuro. El estudio de la comunicación en América Latina. México: FELAFACS.

y Enrique E. SÁNCHEZ RUIZ (1989): Algunas condiciones para la investigación científica de la comunicación en México. Huella No 17, Guadalajara: ITESO.

Gago Huguet Antonio (1992): "Ejes de la Reforma: calidad y pertinencia". Universidad Futura Núm.10, México.

INTERCOM (1988): Estudo Comparativo dos Sistemas de Comunicação Social no Brasil e no México. Projeto de Pesquisa, São Paulo.

LINS DA SILVA Carlos Eduardo (1991): "Industria de la Comunicación: personaje principal de las elecciones presidenciales brasileñas de 1989". Diá-logos de la Comunicación Núm. 29, pp. 56-59. Lima: FELAFACS.

LOPES María Immacolata V. de (1990): Pesquisa em Comunicação: Formulação de um Modelo Metodológico. São Paulo: Loyola. 
- (1991): "La investigación sobre comunicación en Brasil". Comunicación y Sociedad Núm. 10-11, pp. 167-191. Guadalajara.

— D. A. PoBlacion y S.C. DA VIA (1992): O mercado de trabalho em Comunicaçoese Artes e os profissionais formados pela ECA nas décadas de $70 e$ 80. São Paulo: ECA/USP.

LÓPEZ VENERONI Felipe N. (1991): "La comunicación como vacío académico". Diá-logos de la Comunicación Núm 31, pp. 15-24. Lima: FELAFAcs.

MARQues de Melo José (1991a): Comtnicação e Modemidade. O Ensino e a Pesquisa nas Escolas de Comunicação. São Paulo: Loyola.

(1991b): "¿Modernidad o anacronismo? el dilema de las escuelas de comunicación en Brasil". Diá-logos de la Comunicación Núm. 31, pp.49-54. Lima: FELAFACS.

- (Coord) (1992): Comunicación Latinoamericana: Desafios de la Investigación para el Siglo XXI. São Paulo: ALAIC.

MARTín BARBero Jesús (1990a): "Comunicación, campo cultural y proyecto mediador”. Diá-logos de la Comunicación Núm. 26, pp. 6-15. Lima: FELAFACS.

(1990b): "Teoría/Investigación/Producción en la enseñanza de la comunicación”. Diá-logos de la Comunicación Núm. 28, pp. 70-76. Lima: FELAFACS.

(1992): "Pensar la sociedad desde la comunicación: un lugar estratégico para el debate a la modernidad". Diá-logos de la Comunicación Núm. 32, pp.28-33. Lima: FELAFACS.

MCANANY Emile G. (1992): "Cooperación de investigación crítica para Latinoamérica y los Estados Unidos en una era de globalización de la comunicación". En José MARQues De MElo (Coord.) Comunicación Latinoamericana: Desafios de la Investigación para el Siglo XXI. São Paulo: ALAIC. 
MINISTERIO DE EDUCAÇão (1987): Diagnóstico das Escolas de Comunicação Social. Brasilia.

MOREIRA Sonia Virginia (1993): "Los medios contra la corrupción”. Chasqui Núm. 44, pp. 12-14. Quito: CIESPAL.

NEIRA B. Walter (1990): "Los nuevos términos de la democracia y la enseñanza de la comunicación en América Latina". Diá-logos de la Comunicación Núm. 27, pp. 109-117. Lima: FELAFACS.

NIXON Raymond M. (1974): "La enseñanza del periodismo en América Latina". Comunicación y Cultura Núm. 2, pp. 197-212. Buenos Aires.

(1981): Education for Journalism in Latin America: a Report of Progress. Minneapolis: Minnesota Journalism Center.

QUIROZ Teresa (1991): "Los comunicadores sociales, ientre la crítica y el mercado?”. Diá-logos de la Comunicación Núm. 31, pp. 43-48. Lima: FELAFACS.

RoJAs Z. Alberto (1983): "La formación y la práctica profesional de los recursos humanos de la comunicación social”. Seminario de Comunicación Social, pp .83-114. México: AMIC/UAM-A.

SÁNCHEZ GARCÍA Joaquín (1991): "Escuelas de Comunicación ipara qué? Diez años de FELAFACS”. Diá-logos de la Comunicación Núm. 31, pp. 9-14. Lima: FELAFACS.

SÁNCHEZ RuIZ Enrique E. (1992): Medios de Difusión y Sociedad. Notas críticas y Metodológicas. Guadalajara: CEIC/Universidad de Guadalajara.

Solís L. Beatriz (1983):. "Notas sobre la enseñanza de la comunicación”. Connotaciones Núm 4, pp. 53-61. México.

TORQUATO Gaudencio (1993): “Ambientando la destitución de un presidente". Chasqui Núm. 44, pp.10-11. Quito: CIESPAL. 